\title{
Review
}

\section{Handling Macromolecule Signals in the Quantification of the Neurochemical Profile}

\author{
Cristina Cudalbu ${ }^{\mathrm{a}, *}$, Vladimir Mlynárik ${ }^{\mathrm{a}}$ and Rolf Gruetter ${ }^{\mathrm{a}, \mathrm{b}}$ \\ ${ }^{a}$ Laboratory for Functional and Metabolic Imaging, Ecole Polytechnique Fédérale de Lausanne, Lausanne, \\ Switzerland \\ ${ }^{\mathrm{b}}$ Departments of Radiology, Universities of Lausanne and Geneva, Lausanne, Switzerland
}

Accepted 4 April 2012

\begin{abstract}
In vivo localized proton magnetic resonance spectroscopy $\left({ }^{1} \mathrm{H}\right.$ MRS) became a powerful and unique technique to non-invasively investigate brain metabolism of rodents and humans. The main goal of ${ }^{1} \mathrm{H}$ MRS is the reliable quantification of concentrations of metabolites (neurochemical profile) in a well-defined region of the brain. The availability of very high magnetic field strengths combined with the possibility of acquiring spectra at very short echo time have dramatically increased the number of constituents of the neurochemical profile. The quantification of spectra measured at short echo times is complicated by the presence of macromolecule signals of particular importance at high magnetic fields. An error in the macromolecule estimation can lead to substantial errors in the obtained neurochemical profile. The purpose of the present review is to overview methods of high field ${ }^{1} \mathrm{H}$ MRS with a focus on the metabolite quantification, in particular in handling signals of macromolecules. Three main approaches of handling signals of macromolecules are described, namely mathematical estimation of macromolecules, measurement of macromolecules in vivo, and direct acquisition of the in vivo spectrum without the contribution of macromolecules.
\end{abstract}

Keywords: In vivo short echo time ${ }^{1} \mathrm{H}$ MRS, macromolecule contribution, quantification of neurochemical profile

\section{INTRODUCTION}

Over the last decades, in vivo localized proton magnetic resonance spectroscopy $\left({ }^{1} \mathrm{H}\right.$ MRS) became a powerful and unique technique to non-invasively and longitudinally investigate brain metabolism of rodents and humans [1-10]. Proton MRS is one of the most sensitive NMR techniques due to both high gyromagnetic ratio and high natural abundance of ${ }^{1} \mathrm{H}$ nuclei. In addition, nearly all brain metabolites contain protons, so that an important number of biologically relevant metabolites can be observed [11]. ${ }^{1} \mathrm{H}$ MRS

\footnotetext{
*Correspondence to: Cristina Cudalbu, PhD, Ecole Polytechnique Fédérale de Lausanne (EPFL), Laboratory for Functional and Metabolic Imaging (LIFMET), Station 6, CHF1 602 (Bâtiment CH), CH-1015 Lausanne, Switzerland. Tel.: +41 21693 0580; Fax: +41 21693 7960; E-mail: cristina.cudalbu@epfl.ch.
}

is in principle sensitive enough to ensure an in vivo detection of brain metabolites at concentration as low as $0.5 \mathrm{mM}$. The availability of very high magnetic field strengths $(\geq 7 \mathrm{~T})$ combined with the possibility of acquiring spectra at very short echo time (TE) $(<10 \mathrm{~ms})$ have dramatically increased the number of in vivo detectable brain metabolites, allowing in animal models and humans the detection of about 20 metabolites (also called the neurochemical profile). They are markers of: myelination/cell proliferation (phosphocholine, glycerophosphocholine, phosphoethanolamine, Nacetylaspartate (NAA), N-acetylaspartylglutamate), energy metabolism (glucose, lactate, creatine, phosphocreatine, alanine), osmoregulation (taurine, myoinositol), neurotransmitter metabolism (glutamate, glutamine, aspartate, $\gamma$-aminobutyrate, glycine), and antioxidants (ascorbate, glutathione) [1-9, 12, 13]. 
Reliable quantification of low concentration metabolites (ascorbate, glycine) has been previously shown [14-18]. In addition, brain metabolite changes have been demonstrated during development [7, 19-23], reflecting functional and structural evolution and regional specificity $[7,16,19,24]$. It has already been shown that brain metabolite concentrations can vary depending on the type of pathology. The usefulness of the neurochemical profile has been demonstrated in many brain disorders, e.g., in hepatic encephalopathy, Alzheimer's, Huntington's, and Parkinson's diseases, acute traumatic brain injury, cancer, dementia, etc. [11, 25-44], to understand underlying metabolic processes and/or to monitor effect of treatment.

\section{Brain metabolites and macromolecules}

Generally, an in vivo ${ }^{1} \mathrm{H}$ MRS spectrum acquired in the brain contains the contribution of numerous resonances originating from low-molecular weight metabolites which are superimposed on signals of high-molecular weight macromolecules (MM). There are two types of the MM signals, those given by nuclei of backbones of macromolecules having severely restricted mobility, and those given by nuclei of outer parts of macromolecules or those of smaller macromolecules having higher mobility. The nuclei of the macromolecular backbones have very short $\mathrm{T}_{2}$ relaxation times and therefore their signals are difficult to detect. However, the signals of large MM can contain useful clinical information in various pathological conditions. Therefore, new experimental schemes, such as the low excitation pulse angle method, can be helpful [45] in measuring these signals. In addition, for detecting the short $\mathrm{T}_{2}$ signals of large molecules having potential clinical relevance, development of new pulse sequence detecting short $\mathrm{T}_{2}$ signals of large macromolecules would be useful. The present review deals with signals of less hindered parts of MM, which are seen in localized ${ }^{1} \mathrm{H}$ spectra of brain measured with relatively short TE. Previous studies $[4$, 46-53] have extensively reported on macromolecular resonances in brain of animals and humans. These resonances have been, in part, assigned to methyl and methylene resonances of amino acids in cytosolic proteins, such as leucine, isoleucine, valine, threonine, alanine, lysine, arginine, glutamate, glutamine, and $\alpha$ methine protons [46]. However, the presence of lipid resonances cannot be fully excluded [46]. Lipid resonances have been reported in different diseases, i.e., in tumors or demyelination $[25,54,55]$. The evaluation of lipid resonances is quite difficult in brain due to potential contamination with extracerebral lipid containing tissues. Overall, approximately 10 components of macromolecular resonances were observed [4, 46-49, 56]: 0.93 ppm (M1), 1.24 ppm (M2), 1.43 ppm (M3), 1.72 ppm (M4), 2.05 ppm (M5), 2.29 ppm (M6), $3.00 \mathrm{ppm}$ (M7), 3.20 ppm (M8), 3.8-4.0 ppm (M9), and $4.3 \mathrm{ppm}$ (M10).

The macromolecular resonances have physical properties different from those of metabolites, allowing their separate measurement in vivo. The longitudinal $\left(\mathrm{T}_{1}\right)$ and transverse $\left(\mathrm{T}_{2}\right)$ relaxation times of $\mathrm{MM}$ are shorter than those of metabolites [4, 48, 57, 58], due to their large molecular weight. The significant difference in $\mathrm{T}_{1}$ relaxation times enables the selective measurement of MM or metabolites using the inversion recovery technique (IR) [59]. In addition, the shorter $\mathrm{T}_{2}$ relaxation times of $\mathrm{MM}$ effectively eliminate their contribution at longer TE. MM are also characterized by increased linewidths compared to those of metabolites. It should be noted that at high magnetic fields the largely field-independent linewidths of MM increasingly approach that of metabolites. The MM are further characterized by a more hindered mobility as well as diffusivity due to their larger molecular size. A previous study [60] reported that MM have an apparent diffusion coefficient (ADC) 10-20 times lower than that of metabolites. This specific property allows separate measurement of their signals by diffusion weighting techniques (DW), which are based on attenuating the metabolite signal with minimal alteration of MM [61]. Moreover, MM have extensive scalar coupling patterns among the resonances $(\sim 7 \mathrm{~Hz})$, which can affect the detection of $\gamma$-aminobutyrate.

\section{Advantages of high magnetic field}

Several groups have studied the potential advantages of increasing magnetic field for ${ }^{1} \mathrm{H}$ MRS [1, 2, 6, 8, 13, $48,62]$. An increase in intrinsic signal-to-noise ratio (SNR) and chemical shift dispersion are the main benefits at high magnetic fields, which are expected to be useful for low concentrated metabolites, strongly overlapped metabolites, and for metabolites having complex J-coupled spectral patterns. The increased SNR can translate into increased precision of the measurement and quantification or it might be traded off for reduced voxel size, while still maintaining a reasonable SNR for reliable quantification. It has also been shown that these benefits lead to an increased precision of metabolite quantification [1, 2, 8, 62]. Despite these advantages, some factors can interfere and can decrease the quantification precision. 
Resonance frequency of nuclear spins is proportional to a magnitude of the static magnetic field $\left(\mathrm{B}_{0}\right)$. When $\mathrm{B}_{0}$ is spatially inhomogeneous, resonance frequency of ${ }^{1} \mathrm{H}$ nuclei is not the same over the measured volume of a tissue, which results in broadening spectral lines and in decreasing spectral resolution and SNR. Magnetic field inhomogeneities increase with the magnetic field because magnetic susceptibility differences between tissues, blood, bone, and air (in $\mathrm{Hz}$ ) are proportional to the static magnetic field, thus leading to increased peak linewidths (in $\mathrm{Hz}$ ). ${ }^{1} \mathrm{H}$ MRS is technically rather challenging in rodent brain due to the small size of the brain, especially if spectra are acquired in a specific brain region, which, in addition, is located close to the interface between the diamagnetic tissue and paramagnetic oxygen in air. The $\mathrm{B}_{0}$ inhomogeneity induced in the brain by the difference in susceptibility on air/tissue interface are scaled with $\mathrm{B}_{0}$, and powerful high order shim systems are necessary to compensate for them. For relative small volumes selected for localized MRS powerful first- and second-order shims are typically sufficient [6, 63-66]. The chemical shift artifact was shown to be increased due to frequency selective localization. The use of stronger gradients can limit this artifact; however, attention has to be paid to eddy currents. Finally, the $\mathrm{T}_{1}$ relaxation times have been shown to increase with magnetic field [48], leading to increased signal saturation for shorter repletion times (TR). This can be overcome by increasing TR, which would increase the total acquisition time or decreasing signal averaging in the same total acquisition time. However, the $T_{1}$ increase of metabolites does not seem to be important at static magnetic fields beyond 9.4 $\mathrm{T}$ [67].

The purpose of the present study is to review high field ${ }^{1} \mathrm{H}$ MRS techniques of metabolite quantification with the main focus on proper handling of macromolecular baseline. We describe three main approaches of handling the MM: 1) Mathematical estimation of macromolecules; 2) Separate measurement of macromolecules in vivo; and 3) Direct acquisition of an in vivo spectrum without the contribution of macromolecules.

\section{QUANTIFICATION}

The main goal of localized in vivo ${ }^{1} \mathrm{H}$ MRS is the reliable quantification of the concentration of known metabolites in a well-defined region of the brain and the extension of the number of quantifiable metabolites. Metabolite concentration ratios are often used as markers of brain metabolite concentration changes. However, absolute metabolite concentrations can be more valuable for an accurate interpretation of molecular mechanism underlining the pathogenesis of neurological diseases.

Accurate and precise quantification of brain metabolites is challenging and depends on: hardware performance (RF system and shim system), pulse sequence design and adjustments (localization, water suppression, outer volume suppression, shimming, TE, repetition time, pulse calibrations, etc.), data processing (estimate of the signal amplitude or peak area), and quantification strategies (conversion into concentration units). Any misadjustment of the above mentioned acquisition parameters can affect the spectral quality leading to unreliable metabolite concentrations (for more details refer to [12]). Important is the choice of data processing, since many algorithms depend on user input which leads to additional inaccuracies. For more details, see [68-75].

\section{Advantages of high field for quantification}

As already mentioned, measurements at a high magnetic field benefit from decreased strong coupling effects, increased spectral dispersion (increased spectral resolution), and higher SNR (increased sensitivity), which likely improve quantification precision and accuracy $[1-9,62]$. The increase in SNR is expected to also be useful for low concentrated metabolites, for strongly overlapping metabolite peaks, or even for the metabolites having complex J-coupled spectral patterns. Moreover, acquisition performed at a short TE enables to obtain high quality spectra with resolved lines of many metabolites [1-5, 7, 9], thus increasing the amount of biochemical information.

Despite the aforementioned advantages of high magnetic fields, quantification still remains difficult due to the complexity of the spectra (many resonances, peak overlap, contribution of macromolecules, and residual water); the potential presence of distorted lineshapes (residual eddy currents, field inhomogeneity); and a variable baseline (MM, lipid signals, distortions due to insufficient water suppression). Among these factors which complicate the quantification, the last two can be in principle eliminated. The distorted lineshapes can be minimized by the use of optimal shimming [76-79], eddy currents compensation [80], and also by using different post-processing algorithms [81]. In addition, the use of well-calibrated localization sequences and outer volume suppression combined with good water suppression can increase the reproducibility of the baseline in ${ }^{1} \mathrm{H}$ spectrum. 


\section{Short echo times}

Short TE in vivo ${ }^{1} \mathrm{H}$ MRS spectra $(\mathrm{TE}=1-20 \mathrm{~ms})$ contain more information due to minimal $\mathrm{J}$-modulation distortions of multiplets of coupled spin systems such as glutamate, glutamine, myo-inositol, glucose, aspartate, alanine, $\gamma$-aminobutyrate, ascorbate, phosphoethanolamine, glycine, and taurine. In addition, the signal loss due to $\mathrm{T}_{2}$ relaxation is reduced and consequently a more precise quantification is expected. At high magnetic field, the $\mathrm{T}_{2}$ relaxation times of metabolites are shorter [48, 82, 83]. The measurement of $\mathrm{T}_{2}$ relaxation times for the $\mathrm{J}$-coupled metabolites is possible, provided that the signal modulation due to J-coupling is taken into account [83]. Therefore, acquisitions at short TE and long TR (fully relaxed spectra) are considered to be a good choice for absolute quantification, since the effects of relaxation are minimized.

\section{Long echo times}

On the other hand, at longer TE (above $20 \mathrm{~ms}$ ), the number of spectral lines, which can be used for quantification, is reduced due to fast $T_{2}$ relaxation and the J-evolution of coupled spin systems. In addition, care has to be taken regarding the absolute quantification of long TE spectra, since the signal intensities are no longer proportional to the metabolite concentrations and correction factors for the $\mathrm{T}_{2}$ relaxation times have to be used. It has to be emphasized that the $T_{2}$ relaxation times can be different for individual nuclei within the same molecule (e.g., $\mathrm{T}_{2}$ relaxation times of the methyl groups are different from those of the $\mathrm{CH}_{2}$ or $\mathrm{CH}$ groups $[83,84])$ and therefore can further complicate the absolute quantification. As a result, in most in vivo ${ }^{1} \mathrm{H}$ MRS studies, only concentrations of NAA, creatine + phosphocreatine, choline, and lactate can be evaluated.

\section{Quantification algorithms}

To account for the complex spectral pattern of metabolites, severe overlap of proton signals of brain metabolites and the presence of low concentrated metabolites, sophisticated approaches for the spectral analysis are required. Metabolite concentrations are usually determined by fitting the measured in vivo ${ }^{1} \mathrm{H}$ MRS spectrum to a linear combination of spectra of individual metabolites (also called the metabolite basis set) (Fig. 1), which makes quantification feasible, especially for low concentration metabolites $[85,86]$. The metabolite basis set can be obtained either by measuring aqueous solutions of pure metabolites or by quantum mechanics simulations, based on the density-matrix formalism [87], using published values of J-coupling constants and chemical shifts [88], and the relevant acquisition parameters. The measurement of aqueous solutions has to be done under the same conditions as the measurement of in vivo data (the same magnetic field, acquisition sequence, timings, temperature, $\mathrm{pH}$ ). Any change in the acquisition of in vivo data has to be taken into account in the basis set by reacquiring the basis set using the new acquisition parameters. This process can sometimes be time demanding. Therefore, quantum mechanic simulations seem to be a faster alternative, but also more flexible and less expensive. The obtained individual spectra of each metabolite can be used without any post-processing [89-92] or they can be first parameterized [93] and then used as prior knowledge. The influence of the simulated and measured basis sets on the metabolite concentrations using QUEST as a quantification algorithm has been shown to be equivalent [70], provided that lineshape corrections are taken into account. The best fitting errors are estimated from the Cramér-Rao lower bounds (CRLB) [85, 94], which are independent of the quantification algorithm and estimated using the assumption that the model function is correct and complete. Other authors [14] have demonstrated the impact on the quantification of a missing but detectable metabolites from the basis set. They used the case of ascorbate in the analysis of spectra in developing brain and showed that the quantification of other metabolites can be systematically affected.

Well-known time and frequency-domain algorithms, based on metabolite basis sets, are currently used for accurate quantification. The most frequently used algorithms for proton spectra quantification are QUEST [89] from jMRUI software working in the time domain; LCModel [91, 92], working in the frequency domain; AQSES [90], working in time domain; and TDFD [95], working in both time and frequency domains. A more detailed description of these algorithms can be found elsewhere [68-75, 89, 90, 93, 95]. Recently, new algorithms have been developed for the quantification of 2D MRS spectra [96].

\section{HANDLING OF MACROMOLECULE RESONANCES}

As already mentioned, the quantification of short TE spectra is complicated by the presence of underlying broad signals of macromolecules. Reliable 


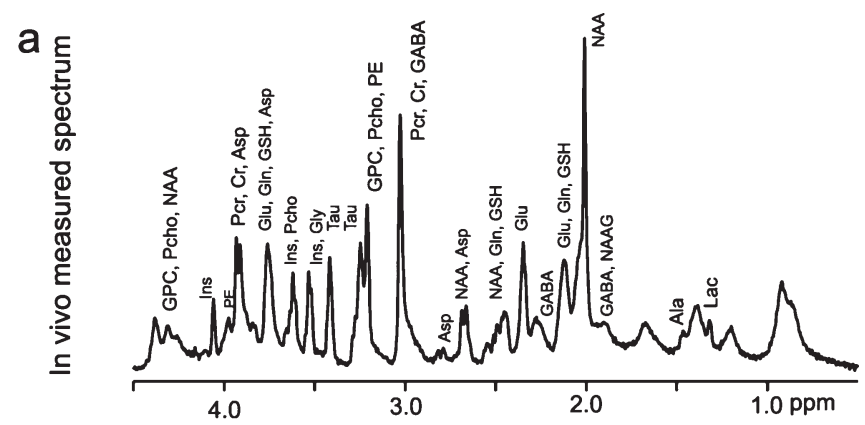

b

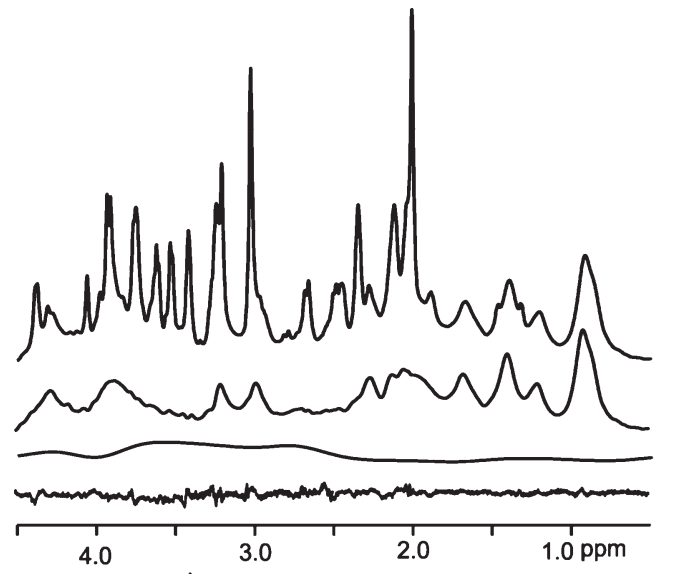

C

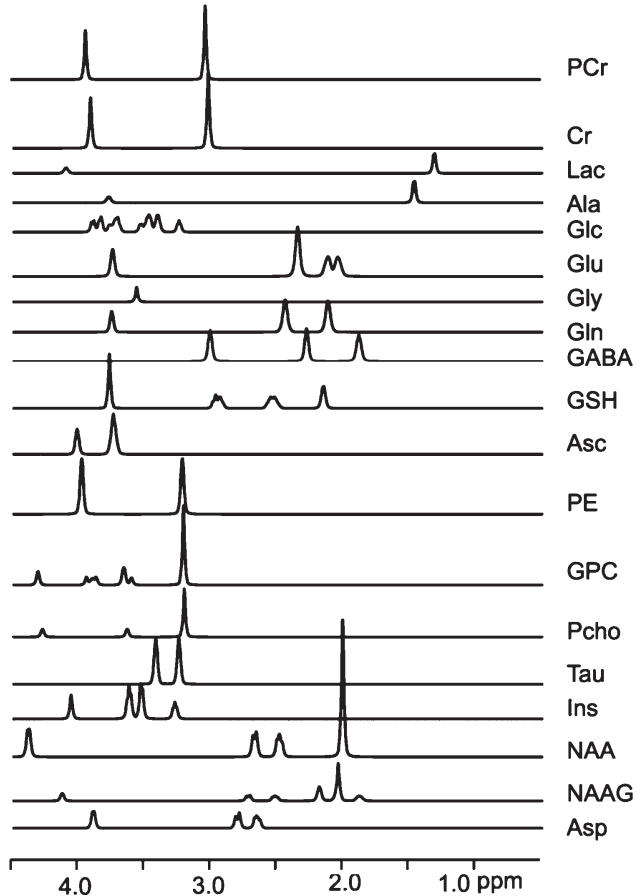

Fig. 1. The LCModel analysis of a representative $14.1 \mathrm{~T}$ spectrum. The measured in vivo spectrum in the rat brain at $14.1 \mathrm{~T}$ is shown in (a). The corresponding LCModel fits using the measured macromolecule spectrum is also displayed in (b). The traces below represent from top to bottom, measured macromolecules, residual baseline and the difference between the measured and fitted data (also called residue of the quantification). The fits of the individual metabolites are plotted in (c). Phosphocholine (PCho), glycerophosphocholine (GPC), phosphoethanolamine (PE), $\mathrm{N}$-acetylaspartate (NAA), $\mathrm{N}$-acetylaspartylglutamate (NAAG), glucose (Glc), lactate (Lac), creatine (Cr), phosphocreatine ( $\mathrm{PCr}$ ), alanine (Ala), taurine (Tau), myo-inositol (Ins), glutamate (Glu), glutamine (Gln), aspartate (Asp), $\gamma$-aminobutyrate (GABA), glycine (Gly), ascorbate (Asc), glutathione (GSH). 
quantification of the MM signals in short TE proton MRS spectra plays a key role in determining the neurochemical profile [4, 68, 69, 71, 97]. An error in the macromolecule estimation can lead to substantial errors in the obtained metabolite concentrations as previously shown $[68,69,71,97]$.

It has already been shown in humans [49] that MM content and composition can depend on the cerebral location and age of the subject, while no gender differences were found. Similarly, Mader and colleagues [98] showed that MM concentration is higher in the cerebellum and motor cortex than in pons or white matter, presumably due to higher content of gray matter in these regions. A recent study performed at $7 \mathrm{~T}$ on healthy volunteers [99] revealed a slight variability in the shape of the MM with a higher concentration for gray matter compared with white matter in the spectral regions around 1.5-1.6 ppm and 2.3-2.6 ppm. Based on the small effects on metabolite concentration of the gray and white matter macromolecule signals, the authors [99] concluded that a general in vivo measured macromolecule spectrum seems sufficient to ensure a reliable quantification of the metabolites in the human brain at $7 \mathrm{~T}$. In fact, the same MM spectrum is commonly used as a part of the basis set in the quantification of spectra from different regions of the brain, regardless the quantification algorithm. Although the metabolite profile regionally varies in the rat brain [20], regional alterations of the $\mathrm{MM}$ signal were reported to be minimal [100, 101], thus a generic experimental macromolecule spectrum acquired in a fairly big voxel (e.g., $4 \times 4 \times 4 \mathrm{~mm}^{3}$ for the rat brain) can be used, provided that the shim quality is sufficient to allow for a good spatial resolution. In addition, few studies have investigated the macromolecular changes with disease $[53,55,102,103]$. One group reported that changes in the macromolecule concentrations can be considered as disease markers [103].

Accounting for the $\mathrm{MM}$ contributions to an in vivo spectrum can be accomplished mainly by using three approaches: 1) Mathematical estimation of macromolecules; 2) Separate measurement of macromolecules in vivo; and 3) Direct acquisition of an in vivo spectrum without the contribution of macromolecules. These three main approaches will be discussed, with the advantages and disadvantages given for each approach.

\section{Mathematical estimation of macromolecules}

When the spectrum of macromolecules is unknown, it can be mathematically approximated. Two kinds of approaches can be distinguished: 1) the macromolecule spectrum included the model function, and 2) the macromolecule spectrum estimated separately in a pre-processing step. Note that both procedures provide a mathematical approximation of the real in vivo macromolecule spectrum $[68,69,71,97]$.

The macromolecule spectrum approximated by a mathematical function (a sum of splines, wavelets, sinusoids or polynomials with adjustable parameters) and then included in the fit [90-92, 104].

This approach is commonly used by several algorithms, e.g., LCModel and AQSES. The importance of the accurate assessment of the macromolecule contributions in quantification of short TE ${ }^{1} \mathrm{H}$ MRS spectra using LCModel was recently demonstrated [69]. In this study, two different approaches of estimating macromolecule contributions in the quantification step at 14.1 $\mathrm{T}$ in the rat brain were compared: mathematical estimation of macromolecules using splines and an acquired macromolecule spectrum in vivo. Despite excellent spectral dispersion at high field, many spectral lines of metabolites have been shown to remain overlapped among each other as well as with MM signals. The LCModel quantification using a basis set of simulated spectra of metabolites combined with the measured spectrum of MM provided an excellent fit to the experimental data. The built-in LCModel spline baselines fitted well the signals of MM, except for the region close to water resonance at around $4.5 \mathrm{ppm}$. However, this smooth approximation of the in vivo MM did not reproduce completely all features of the in vivo spectrum of macromolecules. Compared to the measured MM spectrum, the built-in LCModel spline baseline was elevated around 2.0 and $4.0 \mathrm{ppm}$ and lowered around 3.0 and $3.2 \mathrm{ppm}$, leading to a statistical significant decrease and an increase of concentrations of metabolites resonating in these regions, respectively (Fig. 2). These results showed that an experimental estimation of the macromolecule spectrum can improve the quality of the fit at high field. The absence of the measured macromolecule spectrum in the database leads to a large and unpredictable bias in concentrations of many metabolites (Fig. 2c). Even at the highest magnetic fields available for in vivo experiments, spectral overlap of proton signals from brain metabolites and macromolecules still require an experimental assessment of macromolecular contribution to the proton spectrum for accurate metabolite quantification. Even if the fitted splines reproduce the in vivo macromolecules very well, it was concluded [69] that 


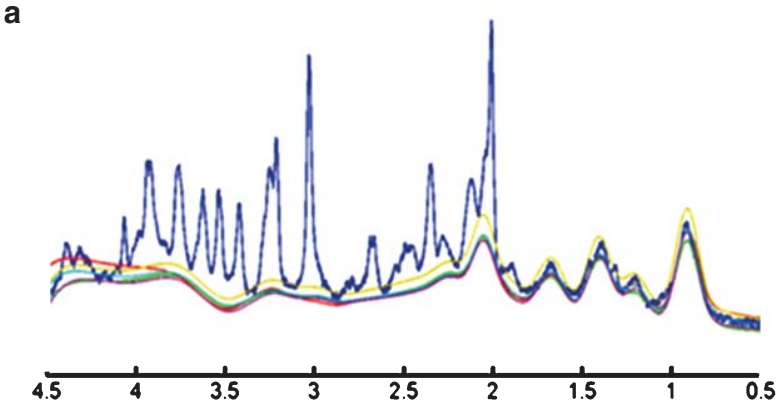

b

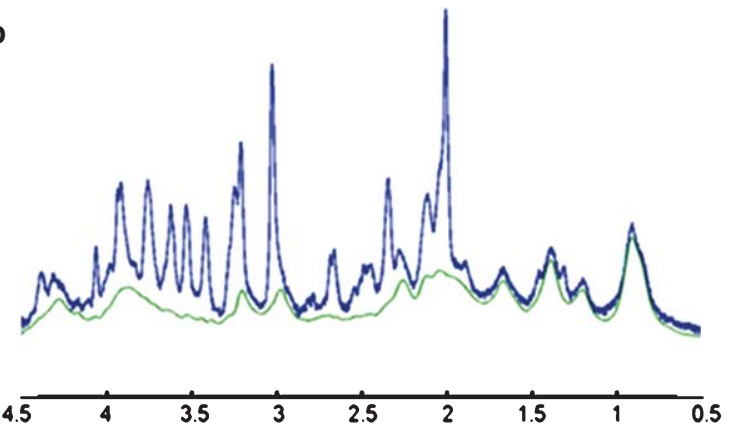

C

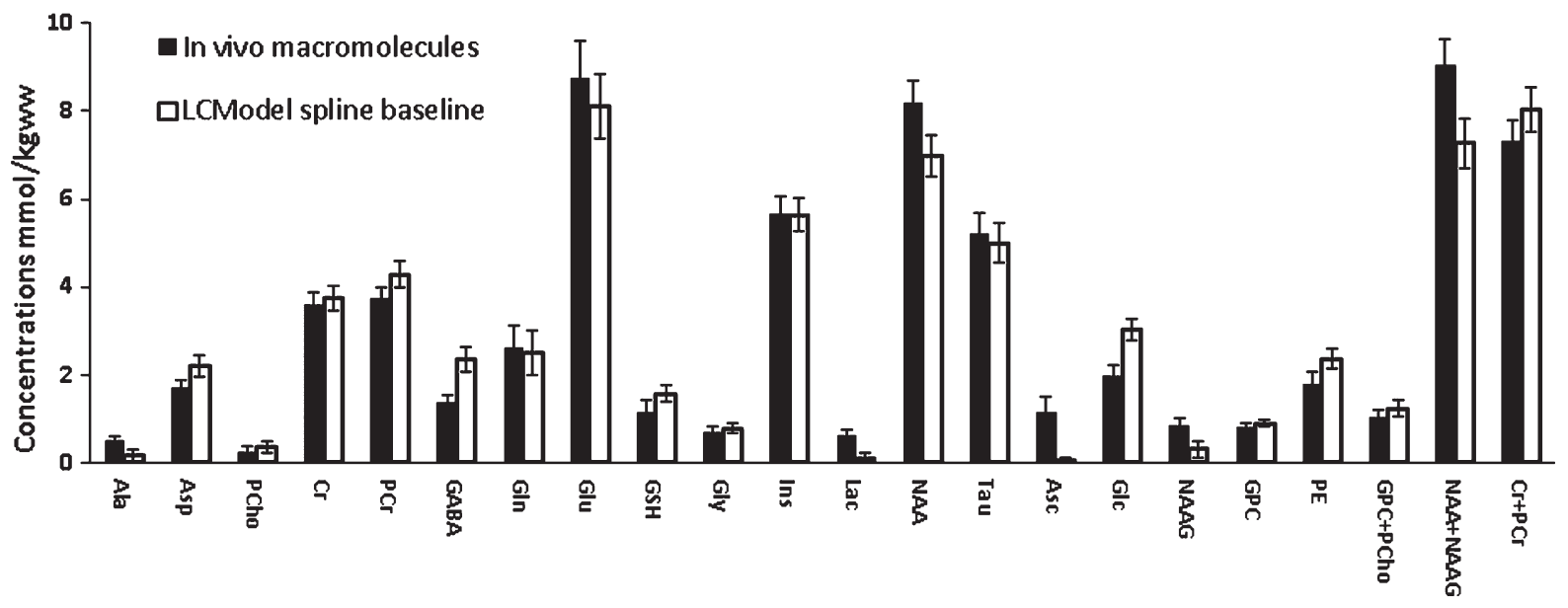

Fig. 2. A representative $14.1 \mathrm{~T}$ spectrum acquired in the rat brain combined with (a) the built-in LCModel spline baseline (the colored lines represent the spline baselines obtained from five different rats and overlaid in different colors) and (b) the measured in vivo macromolecule spectrum. c) Quantification results obtained using the spectrum of macromolecules measured in vivo (black bars) and the built-in LCModel spline baseline (light blues bars).

the measured macromolecular "baseline" represents additional prior knowledge, which led to a more accurate and reliable quantification, especially important at higher static magnetic fields.

\section{The macromolecule spectrum separately}

estimated in a pre-processing step

This can be achieved by: (a) modeling of the MM in the frequency domain with wavelets [104, 105] or in the time-domain using singular value decomposition [106] and subsequent subtraction; (b) truncation of initial data points [107]; and (c) 'Subtract' [89] or numerical filter approach [75].

Only few studies at lower magnetic field strengths of $3 \mathrm{~T}$ and $7 \mathrm{~T}[68,71,97]$ compared the simulated and experimentally obtained spectra of $\mathrm{MM}$ in the quantification of in vivo proton spectra, e.g., using the Subtract-QUEST algorithm [89]. In this algorithm, the macromolecule spectrum is mathematically esti- mated from the first data-points of the MRS signal. This approach exploits the fact that the macromolecule signals decay rapidly due to shorter $T_{2}$ relaxation times. Strategic selection of the number of initial data-points can separate the metabolite part from fast decaying parts and thus decorrelate metabolite and nuisance parameters [89]. In the version of SubtractQUEST used by $[68,71,97]$, the number of data-points involved was chosen by user in an empirical way such that the phased real-part of the MM spectrum estimates was positive and the main resonances of the MM were well identified. When the number of chosen initial data-points was too low, the MM estimates were underestimated, whereas too many initial datapoints led to an overestimation of the MM estimates. As for the LCModel quantification using splines, significant differences in the calculated concentrations were obtained when using the mathematical estimation of the MM. Moreover, it was also observed 
that the macromolecule spectrum estimated by Subtract-QUEST displayed only broader features, inconsistent with what was actually seen in the measured spectra.

Mathematical approaches generally exploit the short $\mathrm{T}_{2}$ relaxation of macromolecules to provide a mathematical approximation of the real in vivo macromolecule spectrum [69, 71, 97, 108]. However at higher magnetic fields, where the macromolecule spectrum is better resolved, additional prior knowledge is needed to obtain an accurate estimation of the metabolite concentrations. The main reason of the poor mathematical estimation of MM at high magnetic fields is that the largely field-independent linewidths of the signals of macromolecules increasingly approaches those of metabolites. Otazo et al. [52] have recently shown a change in the shape in the macromolecule spectrum (in the region of $0 \mathrm{ppm}$ to $3.2 \mathrm{ppm}$ ) when increasing the magnetic field strength from $1.5 \mathrm{~T}$ to $7 \mathrm{~T}$ : at $1.5 \mathrm{~T}$ the macromolecule spectrum was characterized by the presence of 4 broad resonances while at $7 \mathrm{~T}$ the number of resonances increased to 9 .

Despite the problems encountered at a high magnetic field, mathematical modeling of the MM spectrum may be sufficient at low magnetic fields because components of this spectrum are relatively broad compared to the linewidths of the metabolite peaks. Overall, a good mathematical estimation should reproduce well the features of the in vivo macromolecule spectrum, independently of the magnetic field or the quantification algorithm. A recent study [109] showed that the built-in spline estimation of MM using LCModel reproduced well the features of the in vivo measured MM spectrum and provided a good approximation of the MM baseline at $3 \mathrm{~T}$. The experimentally measured MM baseline and the built-in spline baseline gave a similar neurochemical profile, however, small but significant differences in the quantification of some metabolites, such as $16 \%$ for glutamate, were observed. In addition, the adaptability of the built-in spline baseline likely allows a more accurate LCModel quantification especially in pathological cases where the MM can be specifically altered depending on the studied region and the stage of the disease. In such a case, using builtin splines for modeling MM spectrum would largely eliminate the need to measure the MM baseline in each patient. The main advantage of the mathematical approach is a reduction of the total scanning time since measuring the MM spectrum is not necessary for each patient. This feature is important for clinical examination and also during longitudinal/dynamic data acquisition.

\section{Separate measurement of macromolecules in vivo}

The second approach providing the necessary prior knowledge is based on a separate acquisition of the in vivo macromolecule spectrum using methods that exploit the different physical properties of the MM: short $\mathrm{T}_{1}$ compared with the metabolites or the slower diffusion.

Several techniques have been proposed to separately measure the MM spectrum, some of which will be described.

\section{Inversion-recovery $(I R)$ technique}

The IR sequence is considered to be the gold standard for MM acquisition. In general, for the in vivo acquisition of the MM, an IR module consisting of a $180^{\circ} \mathrm{RF}$ pulse and an inversion time (TI) is applied before the localization sequence. The $180^{\circ} \mathrm{RF}$ pulse inverts the $\mathrm{Z}$ magnetization which is being restored toward the equilibrium value during the TI delay. Due to the shorter $\mathrm{T}_{1}$ relaxation time of $\mathrm{MM}$ compared to metabolites, metabolites are nulled at a specific TI, whereas the MM is almost fully recovered. After the excitation pulse, a "metabolite-nulled spectrum" is obtained. However, small residuals of peaks of metabolites are still observed in the metabolite-nulled spectrum due to variability in longitudinal relaxation times of metabolites [48, 67]. Thus, knowledge of the $\mathrm{T}_{1}$ relaxation times of metabolites $[48,67,82,110]$ is required for identification of these residual metabolite signals and subsequent removal by post-processing, typically using HLSVD [111]. In addition, reducing the repetition time in the IR experiment is beneficial for the measurement of MM by reducing the effect of differences in $\mathrm{T}_{1}$ relaxation times of metabolites [8, 112].

Some examples for measuring in vivo MM spectrum using the IR technique at ultra-high magnetic field in rat brain can be found in [2, 4, 69]. For example [3], the SPECIAL sequence was extended with a 2-ms nonselective hyperbolic secant inversion pulse (a bandwidth of $10 \mathrm{kHz}$ ) for the in vivo measurement of macromolecule resonances [2, 69]. A series of IR spectra (TE of $2.8 \mathrm{~ms}$, TR of $2500 \mathrm{~ms}$ and 640 averages) were measured with TI of 420, 600, 750, 850, and $1000 \mathrm{~ms}$ (Fig. 3). Based on the evolution of the metabolite intensities over the series of inversion recovery spectra (changing from negative to positive), the spectrum at $\mathrm{TI}=750 \mathrm{~ms}$ and $\mathrm{TE}=2.8 \mathrm{~ms}$ was taken as a basis for the spectrum of MM, since it contained the smallest residual metabolite peaks (positive or negative). Since $T_{1}$ relaxation times of metabolite 


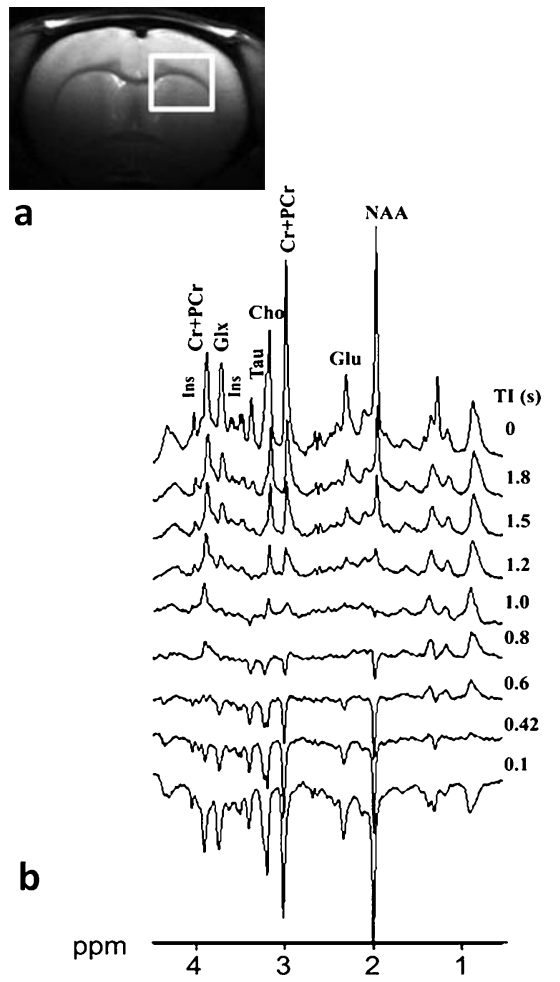

C

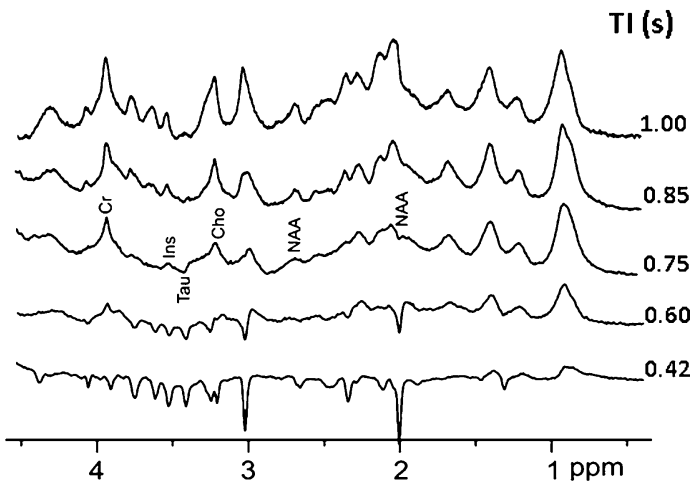

d

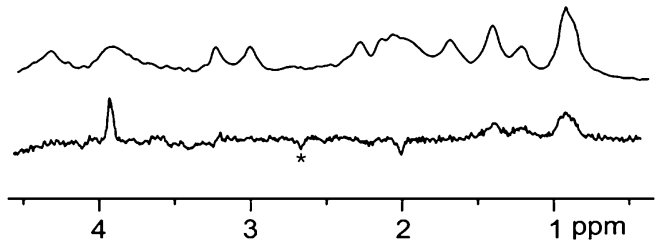

Fig. 3. a) Coronal view of a rat brain showing a typical position of a volume of interest. b) One series of in vivo spectra acquired at $14.1 \mathrm{~T}$ in the rat brain with different inversion times (TI), ranging from 0 to $1.8 \mathrm{~s}$. c) Estimation of the spectrum of macromolecules based on IR-SPECIAL spectra using from bottom to top TIs of $420,600,750,850,1000 \mathrm{~ms}$ ( $\mathrm{TE}=2.8 \mathrm{~ms}$ ). The signals marked on the figure were identified as metabolite residuals in the spectrum at long TE $(40 \mathrm{~ms})$ and consequently removed by HLSVD. d) The spectrum measured with the inversion time of $750 \mathrm{~ms}$, $\mathrm{TE}=40 \mathrm{~ms}$ (bottom) and the final spectrum of macromolecules after removing the residual peaks of metabolites by HLSVD (top). The residual signal of NAA at $2.7 \mathrm{ppm}$ marked with asterisk in the spectrum is reduced and inverted relative to that in the spectrum (c) due to J-evolution.

resonances are not identical $[48,67]$, a macromolecule spectrum with completely suppressed metabolite resonances could not be obtained. Therefore, to confirm the residual metabolite peaks, an inversion recovery spectrum with the same $\mathrm{TI}=750 \mathrm{~ms}$, but with a longer $\mathrm{TE}=40 \mathrm{~ms}$, was acquired (Fig. 3d). In this spectrum the residual signals of metabolites were still visible due to longer $\mathrm{T}_{2}$ relaxation times, while the signals of MM were substantially reduced due to their short $T_{2}$ relaxation times. The residual peaks of NAA at 2.0 and $2.7 \mathrm{ppm}$, taurine at $3.4 \mathrm{ppm}$, total choline at $3.2 \mathrm{ppm}$, myo-inositol at $3.6 \mathrm{ppm}$, and total creatine at $3.9 \mathrm{ppm}$ identified in the spectrum were then removed from the macromolecule spectrum using HLSVD. Care has to be taken when using HLSVD since the user defines manually in the spectrum the frequency region from where the signals to be removed without any additional prior knowledge. Consequently, the metabolite residuals might not be completely removed as any constraints or prior knowledge can be set leading to overor under-estimations of MM and baseline distortions that are likely to affect the metabolite quantification accuracy. A recent study [101] developed a novel and more accurate method than HLSVD for removing residual metabolite signals from the MM spectrum by including advanced prior knowledge on the metabolite residuals. Constraints were set manually on the peak frequency, phase, linewidth, and amplitude to fit the residual metabolite peaks to be removed. The method was combined with AMARES quantification algorithm [113].

\section{Multiple inversion-recovery (MIR) technique}

As already mentioned, the MM spectrum measured using single IR always contained residual of metabolites due to their variation in $\mathrm{T}_{1}$ relaxation times. Consequently, based on the MIR technique proposed by Dixon et al. [114], it was shown that using double or multiple inversion recovery sequences the longitudinal magnetization of the metabolites was close to zero, while the shorter $\mathrm{T}_{1}$ of $\mathrm{MM}$ led to greater signal recovery $[48,56]$. This technique is very similar 
to the IR technique mentioned above with the exception that several IR pulses are used with optimized time delays between them aiming for a better nulling of the metabolite residuals. It was reported that this technique is relatively insensitive to metabolite $T_{1} \mathrm{~S}$ $[48,56]$. However, this method has the drawback that signal intensities in the macromolecule spectrum can be weighted by $T_{1}$ relaxation of individual molecular components, leading to a distorted MM spectrum.

\section{Diffusion weighted spectroscopy (DW MRS)}

The MM are ascribed to cytosolic proteins [46, 47] and hence expected to have a slow self-diffusion constant, which can be assessed using diffusion weighted and measuring the ADC. Indeed MM have been shown to have ADCs 10 to 20 times lower than those of metabolites [60]. Therefore, the use of DW techniques could improve the MM estimation by attenuating the metabolites signal without significantly altering that of MM. The measurement of MM using DW MRS has been proposed by Kunz et al. [61] in the rat brain. By combining IR with DW, the authors showed that the metabolite-nulled spectrum (acquired using only IR) previously contaminated by residuals attributed to creatine, myo-inositol, taurine, choline, NAA as well as glutamine and glutamate peaks, showed significant attenuation (approx 50\%) of these peaks when combining IR with DW, while the MM signals were almost unaffected (Fig. 4). It was thus likely that the final spectrum obtained was composed of only the MM signals with metabolite signals reduced to noise level. The authors concluded that the present technique improves the state-of-art by determining the MM signals purely experimentally, eliminating the need for any post-processing. This technique provides an excellent assessment of the MM with negligible contamination with residual metabolite peaks.

\section{Saturation-recovery (SR) technique}

Hofmann and colleagues [49] have presented a method based on a series of saturation recovery scans on a human scanner at $1.5 \mathrm{~T}$, that allowed for the simultaneous measurement of the MM and the fully relaxed metabolites spectrum. Overall, the authors used for each spectrum $N=16$ different saturation time delays, from 0.4 to $9.0 \mathrm{~s}$. The resulting spectra were modeled point-by-point to a saturation recovery curve of $\mathrm{m}(m \leq \mathrm{N}-1)$ components with predefined $\mathrm{T}_{1}$ values. An arbitrary threshold was chosen (at $500 \mathrm{~ms}$ ) to separate metabolites from MM, then with appropriate weighting of the original SR spectra, fully relaxed $\mathrm{MM}$ and metabolites spectra were calculated without

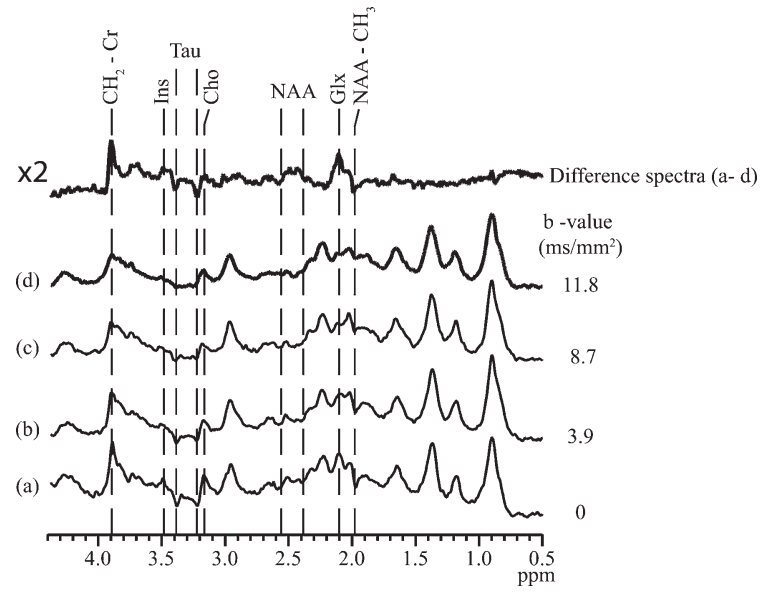

Fig. 4. DW spectra combined with IR acquired with STEAM sequence $(\mathrm{TE}=8 \mathrm{~ms}, \mathrm{TM}=20 \mathrm{~ms}, \mathrm{TR}=2.5 \mathrm{~s}, \mathrm{TI}=740 \mathrm{~ms}, 720$ averages). Diffusion gradient were gradually increased, giving b-value ranging from 0 (a) to $11.8 \mathrm{~ms} / \mu \mathrm{m}^{2}$ (d). The difference spectra between (a) and (d) have a very flat baseline, demonstrating the insensitivity of macromolecules to diffusion weighting experiment. Additionally, several metabolite residuals can be identified in the difference spectra such as creatine $(\mathrm{Cr})$, myo-inositol (Ins), taurine (Tau), N-acetylaspartate (NAA), and glutamine and glutamate compounds (Glx)

further assumptions. Comparing to the IR technique, the $S R$ technique was less susceptible to $T_{1}$ differences. Consequently, the proposed technique allowed good separation between MM and metabolites based on $\mathrm{T}_{1}$ differences.

It is widely accepted that the inclusion of the measured MM spectrum in quantification will lead to improved reliability and accuracy of metabolite concentrations especially at high magnetic fields. For minimizing intensity of residual peaks of metabolites, parameters of the pulse sequence used for the measurement of the MM spectrum should be carefully adjusted. Identification of the residual peaks of metabolites is usually based on different physical properties of macromolecule and metabolite peaks, e.g., on differences in $\mathrm{T}_{1}$ relaxation times when using the IR technique. Residual metabolite peaks have to be unambiguously identified for their subsequent elimination using different post-processing techniques. The postprocessing step has to be done carefully; otherwise it might lead to biased results. Overall, the in vivo measurement of MM can be time demanding, which might be a limiting factor in some studies, e.g., of patients in serious conditions.

Once acquired (either by IR, DW, or SR), the MM spectrum, free of extra cerebral lipids, has then to be 
included in the basis set. As previously mentioned for the metabolites basis set, the MM spectrum can be handled in two ways: 1) can be added to the basis set just as any other metabolite. When included in the basis set, the MM spectrum can be included as a whole [68, $69,71,108]$ or can be first split into separate components [53]. The use of individual components of the MM spectrum can be advantageous when some resonances of the MM spectrum can be affected by diseases; however, the degrees of freedom in the modeling will increase, which might reduce the robustness of the fitting. An alternative approach is to use the whole macromolecule spectrum in the basis set plus some macromolecule/lipid peak components [28] in the regions where the $\mathrm{MM}$ and/or lipid signals are altered by a disease; and 2) can be subtracted from the in vivo signal $[97,115,116]$. The main advantage in subtracting the MM signal from the in vivo signal before quantification is that no correlation between the MM and the metabolites will exist anymore. However, this method will lead to an increase in the noise of the final signal, which will lead to an increase in CRLB of the calculated metabolite concentrations [116]. Consequently, it is advantageous to subtract only the initial macromolecule data-points whose contribution exceeds the noise level.

\section{Direct acquisition of the in vivo metabolite spectrum without the contribution of macromolecules}

Several techniques have been proposed to acquire the metabolite signals without the contributions of MM.

One of them is the acquisition at long TE. This approach exploits the fact that the MMs have shorter $\mathrm{T}_{2}$ than the metabolites. As a result, the acquired spectrum at long TE will not contain the MM contributions anymore. Care has to be taken regarding the absolute quantification of long TE spectra since corrections for the $T_{2}$ relaxation times are necessary. However, in pathologic conditions when the MM or lipids content is increased, spectra can still be contaminated by the presence of $\mathrm{MM}$ as well as lipids.

The multiple IR technique is generally used for the acquisition of the MM spectra. However, by choosing proper time delays between the inversion pulses and a proper repetition time, this technique can also be used for the acquisition of the metaboliteonly spectrum without the MM contributions [117]. In such a spectrum, the short $\mathrm{T}_{1}$ components of macromolecule resonances are saturated, whereas the metabolite peaks having longer $\mathrm{T}_{1}$ relaxation times are almost unaffected.

\section{CONCLUSIONS}

In the present paper we focused on an important aspect of the acquisition of the neurochemical profile, i.e., handling the signals of macromolecules. We reviewed three main approaches of handling signals of MM. Overall, it is well accepted that adding in vivo measured MM spectrum in the quantification step will improve the reliability and accuracy of metabolite concentrations, especially at high magnetic fields where individual MM resonances are better resolved. Finally, the choice of approach to be used will depend substantially on the data under investigation and on the user objectives.

\section{ACKNOWLEDGMENTS}

Supported by Centre d'Imagerie BioMédicale (CIBM) of the UNIL, UNIGE, HUG, CHUV, EPFL, Leenaards and Jeantet Foundations, EU Grant No. MRTN-CT-2006-035801, and the Swiss National Science Foundation (SNF grant 131087). The authors thank to Dr N. Kunz (Laboratory for Functional and Metabolic Imaging (LIFMET), Ecole Polytechnique Fédérale de Lausanne (EPFL), Lausanne, Switzerland) for providing the Fig. 4.

Authors' disclosures available online http://www.jalz.com/disclosures/view.php?id=1247.

\section{REFERENCES}

[1] Mekle R, Mlynarik V, Gambarota G, Hergt M, Krueger G, Gruetter R (2009) MR spectroscopy of the human brain with enhanced signal intensity at ultrashort echo times on a clinical platform at $3 \mathrm{~T}$ and $7 \mathrm{~T}$. Magn Reson Med 61, 1279-1285.

[2] Mlynarik V, Cudalbu C, Xin L, Gruetter R (2008) 1H NMR spectroscopy of rat brain in vivo at 14.1 Tesla: Improvements in quantification of the neurochemical profile. J Magn Reson 194, 163-168.

[3] Mlynarik V, Gambarota G, Frenkel H, Gruetter R (2006) Localized short-echo-time proton MR spectroscopy with full signal-intensity acquisition. Magn Reson Med 56, 965970.

[4] Pfeuffer J, Tkac I, Provencher SW, Gruetter R (1999) Toward an in vivo neurochemical profile: Quantification of 18 metabolites in short-echo-time (1)H NMR spectra of the rat brain. J Magn Reson 141, 104-120.

[5] Tkac I, Andersen P, Adriany G, Merkle H, Ugurbil K, Gruetter R (2001) In vivo $1 \mathrm{H}$ NMR spectroscopy of the human brain at 7 T. Magn Reson Med 46, 451-456.

[6] Tkac I, Gruetter R (2005) Methodology of H NMR spectroscopy of the human brain at very high magnetic fields. Appl Magn Reson 29, 139-157. 
[7] Tkac I, Henry PG, Andersen P, Keene CD, Low WC, Gruetter R (2004) Highly resolved in vivo $1 \mathrm{H}$ NMR spectroscopy of the mouse brain at 9.4 T. Magn Reson Med 52, 478-484.

[8] Tkac I, Oz G, Adriany G, Ugurbil K, Gruetter R (2009) In vivo $1 \mathrm{H}$ NMR spectroscopy of the human brain at high magnetic fields: Metabolite quantification at $4 \mathrm{~T}$ vs. 7 T. Magn Reson Med 62, 868-879.

[9] Tkac I, Starcuk Z, Choi IY, Gruetter R (1999) In vivo $1 \mathrm{H}$ NMR spectroscopy of rat brain at $1 \mathrm{~ms}$ echo time. Magn Reson Med 41, 649-656.

[10] Cudalbu C, Cavassila S, Ratiney H, Grenier D, Briguet A, Graveron-Demilly D (2006) Estimation of metabolite concentrations of healthy mouse brain by magnetic resonance spectroscopy at 7 T. Comptes Rendus Chimie 9, 534-538.

[11] Williams S (1999) Cerebral amino acids studied by nuclear magnetic resonance spectroscopy in vivo. Prog Nucl Magn Reson Spect 34, 301-326.

[12] Kreis R (2004) Issues of spectral quality in clinical $1 \mathrm{H}-$ magnetic resonance spectroscopy and a gallery of artifacts. NMR Biomed 17, 361-381.

[13] Gruetter R, Weisdorf SA, Rajanayagan V, Terpstra M, Merkle H, Truwit CL, Garwood M, Nyberg SL, Ugurbil K (1998) Resolution improvements in in vivo 1H NMR spectra with increased magnetic field strength. J Magn Reson $\mathbf{1 3 5}$, 260-264.

[14] Terpstra M, Rao R, Tkac I (2010) Region-specific changes in ascorbate concentration during rat brain development quantified by in vivo (1)H NMR spectroscopy. NMR Biomed $\mathbf{2 3}$, 1038-1043.

[15] Terpstra M, Ugurbil K, Tkac I (2010) Noninvasive quantification of human brain ascorbate concentration using $1 \mathrm{H}$ NMR spectroscopy at 7 T. NMR Biomed 23, 227-232.

[16] Xin L, Gambarota G, Duarte JM, Mlynarik V, Gruetter R (2010) Direct in vivo measurement of glycine and the neurochemical profile in the rat medulla oblongata. NMR Biomed 23, 1097-1102.

[17] Gambarota G, Mekle R, Xin L, Hergt M, van der Zwaag W, Krueger G, Gruetter R (2009) In vivo measurement of glycine with short echo-time $1 \mathrm{H}$ MRS in human brain at 7 T. MAGMA 22, 1-4.

[18] Terpstra M, Gruetter R (2004) 1H NMR detection of vitamin $\mathrm{C}$ in human brain in vivo. Magn Reson Med 51, 225-229.

[19] Kulak A, Duarte JM, Do KQ, Gruetter R (2010) Neurochemical profile of the developing mouse cortex determined by in vivo $1 \mathrm{H}$ NMR spectroscopy at $14.1 \mathrm{~T}$ and the effect of recurrent anaesthesia. J Neurochem 115, 1466-1477.

[20] Tkac I, Rao R, Georgieff MK, Gruetter R (2003) Developmental and regional changes in the neurochemical profile of the rat brain determined by in vivo $1 \mathrm{H}$ NMR spectroscopy. Magn Reson Med 50, 24-32.

[21] Kreis R, Hofmann L, Kuhlmann B, Boesch C, Bossi E, Huppi PS (2002) Brain metabolite composition during early human brain development as measured by quantitative in vivo $1 \mathrm{H}$ magnetic resonance spectroscopy. Magn Reson Med 48, 949-958.

[22] Kreis R, Ernst T, Ross BD (1993) Development of the human brain: In vivo quantification of metabolite and water content with proton magnetic resonance spectroscopy. Magn Reson Med 30, 424-437.

[23] Bates TE, Williams SR, Gadian DG, Bell JD, Small RK, Iles RA (1989) $1 \mathrm{H}$ NMR study of cerebral development in the rat. NMR Biomed 2, 225-229.

[24] Lei H, Poitry-Yamate C, Preitner F, Thorens B, Gruetter R (2010) Neurochemical profile of the mouse hypothalamus using in vivo $1 \mathrm{H}$ MRS at 14.1 T. NMR Biomed 23, 578-583.

[25] Mlynárik V, Cudalbu C, Clément V, Marino D, Radovanovic I, Gruetter R (2012) In vivo metabolic profiling of glioma-initiating cells using proton magnetic resonance spectroscopy at 14.1 Tesla. NMR Biomed 25, 506-513.

[26] van de Looij Y, Chatagner A, Huppi PS, Gruetter R, Sizonenko SV (2011) Longitudinal MR assessment of hypoxic ischemic injury in the immature rat brain. Magn Reson Med 65, 305-312.

[27] Tkac I, Keene CD, Pfeuffer J, Low WC, Gruetter R (2001) Metabolic changes in quinolinic acid-lesioned rat striatum detected non-invasively by in vivo (1)H NMR spectroscopy. J Neurosci Res 66, 891-898.

[28] Oz G, Tkac I, Charnas LR, Choi IY, Bjoraker KJ, Shapiro EG, Gruetter R (2005) Assessment of adrenoleukodystrophy lesions by high field MRS in non-sedated pediatric patients. Neurology 64, 434-441.

[29] Tkac I, Dubinsky JM, Keene CD, Gruetter R, Low WC (2007) Neurochemical changes in Huntington R6/2 mouse striatum detected by in vivo $1 \mathrm{H}$ NMR spectroscopy. $J \mathrm{Neu}$ rochem 100, 1397-1406.

[30] Cudalbu C, Montavont A, Ryvlin P, Cavassila S (2008) Brain metabolite concentration estimates using Magnetic Resonance Spectroscopy in a chronic model of temporal lobe epilepsy. Comptes Rendus Chimie 11, 434-441.

[31] Berthet C, Lei H, Thevenet J, Gruetter R, Magistretti PJ, Hirt L (2009) Neuroprotective role of lactate after cerebral ischemia. J Cereb Blood Flow Metab 29, 1780-1789.

[32] Cudalbu C, Lanz B, Morgenthaler F, Pilloud Y, Mlynarik V, Gruetter R (2009) Glutamine synthesis rate in the hyperammonaemic rat brain using simultaneous localized in vivo $\mathrm{H}-1$ and N-15 MRS. J Cereb Blood Flow Metab 29, S609-S610.

[33] Rao R, Ennis K, Long JD, Ugurbil K, Gruetter R, Tkac I (2010) Neurochemical changes in the developing rat hippocampus during prolonged hypoglycemia. J Neurochem 114, 728-738.

[34] Berthet C, Lei H, Gruetter R, Hirt L (2011) Early predictive biomarkers for lesion after transient cerebral ischemia. Stroke 42, 799-805.

[35] Duarte JM, Kulak A, Gholam-Razaee MM, Cuenod M, Gruetter R, Do KQ (2011) N-Acetylcysteine normalizes neurochemical changes in the glutathione-deficient schizophrenia mouse model during development. Biol Psychiatry, doi: 10.1016/j.biopsych.2011.07.035.

[36] Just N, Cudalbu C, Lei H, Gruetter R (2011) Effect of manganese chloride on the neurochemical profile of the rat hypothalamus. J Cereb Blood Flow Metab 31, 2324-2333.

[37] Kunz N, Camm EJ, Somm E, Lodygensky G, Darbre S, Aubert ML, Huppi PS, Sizonenko SV, Gruetter R (2011) Developmental and metabolic brain alterations in rats exposed to bisphenol A during gestation and lactation. Int $J$ Dev Neurosci 29, 37-43.

[38] Kreis R, Wingeier K, Vermathen P, Giger E, Joncourt F, Zwygart K, Kaufmann F, Boesch C, Steinlin M (2011) Brain metabolite composition in relation to cognitive function and dystrophin mutations in boys with Duchenne muscular dystrophy. NMR Biomed 24, 253-262.

[39] Kreis R, Ross BD, Farrow NA, Ackerman Z (1992) Metabolic disorders of the brain in chronic hepatic encephalopathy detected with H-1 MR spectroscopy. Radiology 182, 19-27.

[40] Kreis R, Ross BD (1992) Cerebral metabolic disturbances in patients with subacute and chronic diabetes mellitus: Detection with proton MR spectroscopy. Radiology 184, 123-130. 
[41] Kreis R, Pfenninger J, Herschkowitz N, Boesch C (1995) In vivo proton magnetic resonance spectroscopy in a case of Reye's syndrome. Intensive Care Med 21, 266269.

[42] Kreis R, Farrow N, Ross BD (1991) Localized 1H NMR spectroscopy in patients with chronic hepatic encephalopathy. Analysis of changes in cerebral glutamine, choline and inositols. NMR Biomed 4, 109-116.

[43] Kreis R, Farrow N, Ross BD (1990) Diagnosis of hepatic encephalopathy by proton magnetic resonance spectroscopy. Lancet 336, 635-636.

[44] Alf MF, Lei H, Berthet C, Hirt L, Gruetter R, Mlynarik V (2012) High-resolution spatial mapping of changes in the neurochemical profile after focal ischemia in mice. $N M R$ Biomed 25, 247-254.

[45] Mandal PK, Akolkar H (2011) A new experimental approach and signal processing scheme for the detection and quantitation of (3)(1)P brain neurochemicals from in vivo MRS studies using dual tuned $((1) \mathrm{H} /(3)(1) \mathrm{P})$ head coil. Biochem Biophys Res Commun 412, 302-306.

[46] Behar KL, Ogino T (1993) Characterization of macromolecule resonances in the $1 \mathrm{H}$ NMR spectrum of rat brain. Magn Reson Med 30, 38-44.

[47] Behar KL, Rothman DL, Spencer DD, Petroff OA (1994) Analysis of macromolecule resonances in 1H NMR spectra of human brain. Magn Reson Med 32, 294-302.

[48] de Graaf RA, Brown PB, McIntyre S, Nixon TW, Behar KL, Rothman DL (2006) High magnetic field water and metabolite proton $\mathrm{T} 1$ and $\mathrm{T} 2$ relaxation in rat brain in vivo. Magn Reson Med 56, 386-394.

[49] Hofmann L, Slotboom J, Boesch C, Kreis R (2001) Characterization of the macromolecule baseline in localized (1)H-MR spectra of human brain. Magn Reson Med 46, 855-863.

[50] Kauppinen RA, Kokko H, Williams SR (1992) Detection of mobile proteins by proton nuclear magnetic resonance spectroscopy in the guinea pig brain ex vivo and their partial purification. J Neurochem 58, 967-974.

[51] Kauppinen RA, Niskanen T, Hakumaki J, Williams SR (1993) Quantitative analysis of $1 \mathrm{H}$ NMR detected proteins in the rat cerebral cortex in vivo and in vitro. NMR Biomed 6, 242-247.

[52] Otazo R, Mueller B, Ugurbil K, Wald L, Posse S (2006) Signal-to-noise ratio and spectral linewidth improvements between 1.5 and 7 Tesla in proton echo-planar spectroscopic imaging. Magn Reson Med 56, 1200-1210.

[53] Seeger U, Klose U, Mader I, Grodd W, Nagele T (2003) Parameterized evaluation of macromolecules and lipids in proton MR spectroscopy of brain diseases. Magn Reson Med 49, 19-28.

[54] Larsson HB, Christiansen P, Jensen M, Frederiksen J, Heltberg A, Olesen J, Henriksen O (1991) Localized in vivo proton spectroscopy in the brain of patients with multiple sclerosis. Magn Reson Med 22, 23-31.

[55] Howe FA, Barton SJ, Cudlip SA, Stubbs M, Saunders DE Murphy M, Wilkins P, Opstad KS, Doyle VL, McLean MA, Bell BA, Griffiths JR (2003) Metabolic profiles of human brain tumors using quantitative in vivo $1 \mathrm{H}$ magnetic resonance spectroscopy. Magn Reson Med 49, 223-232.

[56] de Graaf RA ed. (1998) In vivo NMR spectroscopy: Principles and techniques. Wiley, University of Virginia, pp. 68-69.

[57] Kreis R, Slotboom J, Hofmann L, Boesch C (2005) Integrated data acquisition and processing to determine metabolite contents, relaxation times, and macromolecule baseline in single examinations of individual subjects. Magn Reson Med 54, 761-768.

[58] Kreis R, Ernst T, Ross BD (1993) Absolute quantitation of water and metabolites in the human brain. 2. Metabolite concentrations. J Magn Reson Ser B 102, 9-19.

[59] Vold R, Waugh J, Klein M, Phelps D (1968) Measurement of spin relaxation in complex systems. J Chem Phys $\mathbf{4 8 ,}$ 3831-3832.

[60] Pfeuffer J, Tkac I, Gruetter R (2000) Extracellularintracellular distribution of glucose and lactate in the rat brain assessed noninvasively by diffusion-weighted $1 \mathrm{H}$ nuclear magnetic resonance spectroscopy in vivo. J Cereb Blood Flow Metab 20, 736-746.

[61] Kunz N, Cudalbu C, Mlynarik V, Huppi PS, Sizonenko SV, Gruetter R (2010) Diffusion-weighted spectroscopy: A novel approach to determine macromolecule resonances in short-echo time 1H-MRS. Magn Reson Med 64, 939-946.

[62] Bartha R, Drost DJ, Menon RS, Williamson PC (2000) Comparison of the quantification precision of human short echo time (1)H spectroscopy at 1.5 and 4.0 Tesla. Magn Reson Med 44, 185-192.

[63] Tkac I (2011) Requirements for reliable metabolite profiling. Proc Intl Soc Magn Reson Med, Morning categorical course, MRS - Metabolite Profiling \& Metabolism.

[64] de Graaf R (2010) How to get meaningful MRS data. Proc Intl Soc Magn Reson Med, Morning categorical course, Potentials \& Challenges of High Field MRS.

[65] Tkac I (2010) Shimming \& MRS. Proc Intl Soc Magn Reson $\mathrm{Med}$, Morning categorical course, Potentials \& Challenges of High Field MRS.

[66] Tkac I (2008) The Ingredients of a Successful MRS Study at Ultra-High-Field. Proc Intl Soc Magn Reson Med, Morning categorical course, Ultra-High-Field MR in Humans.

[67] Cudalbu C, Mlynarik V, Xin L, Gruetter R (2009) Comparison of $\mathrm{T} 1$ relaxation times of the neurochemical profile in rat brain at 9.4 tesla and 14.1 tesla. Magn Reson Med 62, 862-867.

[68] Cudalbu C, Mlynarik V, Xin L, Gruetter R (2008) Comparison of two approaches to model the macromolecule spectrum for the quantification of short TE H-1 MRS spectra. 2008 Ieee Intl Workshop Imag Syst Techn 309-312.

[69] Cudalbu C, Mlynarik V, Xin L, Gruetter R (2009) Quantification of in vivo short echo-time proton magnetic resonance spectra at $14.1 \mathrm{~T}$ using two different approaches of modelling the macromolecule spectrum. Measure Science Technol 20, 104034

[70] Cudalbu C, Cavassila S, Rabeson H, van Ormonde D, Graveron-Demilly D (2008) Influence of measured and simulated basis sets on metabolite concentration estimates. NMR Biomed 21, 627-636.

[71] Cudalbu C, Bucur A, Graveron-Demilly D, Beuf O, Cavassila S (2007) Comparison of two strategies of background-accommodation: Influence on the metabolite concentration estimation from in vivo Magnetic resonance spectroscopy data. Conf Proc IEEE Eng Med Biol Soc 2007, 2077-2080.

[72] Helms G (2008) The principles of quantification applied to in vivo proton MR spectroscopy. Eur J Radiol 67, 218-229.

[73] Mandal PK (2012) In vivo proton magnetic resonance spectroscopic signal processing for the absolute quantitation of brain metabolites. Eur J Radiol 81, e653-e664.

[74] Poullet JB, Sima DM, Van Huffel S (2008) MRS signal quantitation: A review of time- and frequency-domain methods. J Magn Reson 195, 134-144. 
[75] Poullet JB, Sima DM, Van Huffel S (2006) Frequencyselective quantification of short-echo time magnetic resonance spectra. Conf Proc IEEE Eng Med Biol Soc 1, 6351-6355.

[76] Gruetter R (1993) Automatic, localized in vivo adjustment of all first- and second-order shim coils. Magn Reson Med 29, 804-811.

[77] Gruetter R, Ugurbil K, Seaquist ER (2000) Effect of acute hyperglycemia on visual cortical activation as measured by functional MRI. J Neurosci Res 62, 279-285.

[78] Juchem C, Brown PB, Nixon TW, McIntyre S, Rothman DL, de Graaf RA (2011) Multicoil shimming of the mouse brain. Magn Reson Med 66, 893-900.

[79] Juchem C, Nixon TW, McIntyre S, Boer VO, Rothman DL, de Graaf RA (2011) Dynamic multi-coil shimming of the human brain at 7 T. J Magn Reson 212, 280-288.

[80] Terpstra M, Andersen PM, Gruetter R (1998) Localized eddy current compensation using quantitative field mapping. J Magn Reson 131, 139-143.

[81] Bartha R, Drost DJ, Menon RS, Williamson PC (2000) Spectroscopic lineshape correction by QUECC: Combined QUALITY deconvolution and eddy current correction. Magn Reson Med 44, 641-645.

[82] Cudalbu C, Rengle A, Beuf O, Cavassila S (2008) Rat brain metabolite relaxation time estimates using magnetic resonance spectroscopy at two different field strengths. Comptes Rendus Chimie 11, 442-447.

[83] Xin L, Gambarota G, Mlynarik V, Gruetter R (2008) Proton $\mathrm{T} 2$ relaxation time of $\mathrm{J}$-coupled cerebral metabolites in rat brain at 9.4 T. NMR Biomed 21, 396-401.

[84] Marjanska M, Auerbach EJ, Valabregue R, Van de Moortele PF, Adriany G, Garwood M (2012) Localized 1H NMR spectroscopy in different regions of human brain in vivo at $7 \mathrm{~T}$ : T2 relaxation times and concentrations of cerebral metabolites. NMR Biomed 25, 332-339.

[85] Cavassila S, Deval S, Huegen C, van Ormondt D, GraveronDemilly D (2000) Cramer-Rao bound expressions for parametric estimation of overlapping peaks: Influence of prior knowledge. J Magn Reson 143, 311-320.

[86] Cavassila S, Deval S, Huegen C, Van Ormondt D, Graveron-Demilly D (1999) The beneficial influence of prior knowledge on the quantitation of in vivo magnetic resonance spectroscopy signals. Invest Radiol 34, 242-246.

[87] Smith SA, Levante TO, Meier BH, Ernst RR (1994) Computer-simulations in magnetic-resonance - an objectoriented programming approach. J Magn Reson Ser A 106, 75-105.

[88] Govindaraju V, Young K, Maudsley AA (2000) Proton NMR chemical shifts and coupling constants for brain metabolites. NMR Biomed 13, 129-153.

[89] Ratiney H, Sdika M, Coenradie Y, Cavassila S, van Ormondt D, Graveron-Demilly D (2005) Time-domain semi-parametric estimation based on a metabolite basis set. NMR Biomed 18, 1-13.

[90] Poullet JB, Sima DM, Simonetti AW, De Neuter B, Vanhamme L, Lemmerling P, Van Huffel S (2007) An automated quantitation of short echo time MRS spectra in an open source software environment: AQSES. NMR Biomed 20, 493-504.

[91] Provencher SW (1993) Estimation of metabolite concentrations from localized in vivo proton NMR spectra. Magn Reson Med 30, 672-679.

[92] Provencher SW (2001) Automatic quantitation of localized in vivo $1 \mathrm{H}$ spectra with LCModel. NMR Biomed 14, 260264.
[93] Hofmann L, Slotboom J, Jung B, Maloca P, Boesch C, Kreis R (2002) Quantitative 1H-magnetic resonance spectroscopy of human brain: Influence of composition and parameterization of the basis set in linear combination model-fitting. Magn Reson Med 48, 440-453.

[94] Cavassila S, Deval S, Huegen C, van Ormondt D, GraveronDemilly D (2001) Cramer-Rao bounds: An evaluation tool for quantitation. NMR Biomed 14, 278-283.

[95] Slotboom J, Boesch C, Kreis R (1998) Versatile frequency domain fitting using time domain models and prior knowledge. Magn Reson Med 39, 899-911.

[96] Schulte RF, Boesiger P (2006) ProFit: Two-dimensional prior-knowledge fitting of J-resolved spectra. NMR Biomed 19, 255-263.

[97] Gottschalk M, Lamalle L, Segebarth C (2008) Short-TE localised 1H MRS of the human brain at $3 \mathrm{~T}$ : Quantification of the metabolite signals using two approaches to account for macromolecular signal contributions. NMR Biomed 21, 507-517.

[98] Mader I, Seeger U, Karitzky J, Erb M, Schick F, Klose U (2002) Proton magnetic resonance spectroscopy with metabolite nulling reveals regional differences of macromolecules in normal human brain. J Magn Reson Imaging 16, 538-546

[99] Schaller B, Xin L, Gruetter R (2012) Influence of tissue specific macromolecule baseline on the metabolite quantification in human brain at 7 Tesla. Proc Intl Soc Magn Reson Med, 1774.

[100] Xin L, Mlynárik V, Lei H, Gruetter R (2010) Influence of regional macromolecule baseline on the quantification of neurochemical profile in rat brain. Proc Intl Soc Magn Reson Med 18, 321.

[101] Craveiro M, Cudalbu C, Gruetter R (2012) Regional alterations of the brain macromolecule resonances investigated in the mouse brain using an improved method for the preprocessing of the macromolecular signal. Proc Intl Soc Mag Reson Med, 1748.

[102] Graham GD, Hwang JH, Rothman DL, Prichard JW (2001) Spectroscopic assessment of alterations in macromolecule and small-molecule metabolites in human brain after stroke. Stroke 32, 2797-2802.

[103] Mader I, Seeger U, Weissert R, Klose U, Naegele T, Melms A, Grodd W (2001) Proton MR spectroscopy with metabolite-nulling reveals elevated macromolecules in acute multiple sclerosis. Brain 124, 953-961.

[104] Soher BJ, Young K, Maudsley AA (2001) Representation of strong baseline contributions in 1H MR spectra. Magn Reson Med 45, 966-972.

[105] Young K, Soher BJ, Maudsley AA (1998) Automated spectral analysis II: Application of wavelet shrinkage for characterization of non-parameterized signals. Magn Reson Med 40, 816-821.

[106] Coenradie Y, de Beer R, van Ormondt D, Cavassila S, Ratiney H, Graveron-Demilly D (2002) Background signal parametrization in vivo MR spectroscopy. MAGMA 15, 369.

[107] Stanley JA, Pettegrew JW (2001) Postprocessing method to segregate and quantify the broad components underlying the phosphodiester spectral region of in vivo (31)P brain spectra. Magn Reson Med 45, 390-396.

[108] Cudalbu C, Beuf O, Cavassila S (2009) In vivo short echo time localized H-1 MRS of the rat brain at $7 \mathrm{~T}$ : Influence of two strategies of background-accommodation on the metabolite concentration estimation using QUEST. J Signal Process Syst Signal Image Video Technol 55, 25-34. 
[109] Schaller B, Xin L, Gruetter R (2012) Quantification differences of $1 \mathrm{H}$ spectra in human brain at 3 Tesla using the acquired macromolecule baseline or the built-in LCModel spline baseline. Proc Intl Soc Magn Reson Med, 1780.

[110] Mlynarik V, Gruber S, Moser E (2001) Proton T (1) and T (2) relaxation times of human brain metabolites at 3 Tesla. NMR Biomed 14, 325-331.

[111] Pijnappel WWF, van den Boogaart A, de Beer R, van Ormondt D (1992) SVD-based quantification of magnetic resonance signals. J Magn Reson 97, 122-134.

[112] Oz G, Tkac I (2011) Short-echo, single-shot, full-intensity proton magnetic resonance spectroscopy for neurochemical profiling at $4 \mathrm{~T}$ : Validation in the cerebellum and brainstem. Magn Reson Med 65, 901-910.

[113] Vanhamme L, van den Boogaart A, Van Huffel S (1997) Improved method for accurate and efficient quantification of MRS data with use of prior knowledge. J Magn Reson 129, 35-43.
[114] Dixon WT, Sardashti M, Castillo M, Stomp GP (1991) Multiple inversion recovery reduces static tissue signal in angiograms. Magn Reson Med 18, 257-268.

[115] Kassem MN, Bartha R (2003) Quantitative proton shortecho-time LASER spectroscopy of normal human white matter and hippocampus at 4 Tesla incorporating macromolecule subtraction. Magn Reson Med 49, 918-927.

[116] Rabeson H, Ratiney H, Capobianco E, de Beer R, van Ormondt D, Graveron-Demilly D (2007) Is spending extra scan time on measuring a 'macromolecules-only' signal worthwhile? Proc Intl Soc Magn Reson Med 15, 1392.

[117] Knight-Scott J (1999) Application of multiple inversion recovery for suppression of macromolecule resonances in short echo time (1)H NMR spectroscopy of human brain. $J$ Magn Reson 140, 228-234. 